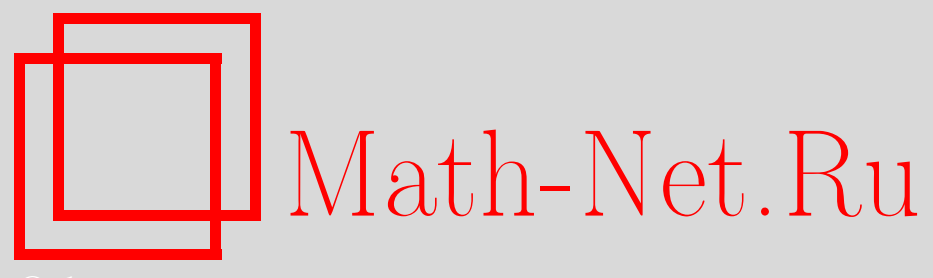

Р. В. Богонатов, Максимальные и минимальные представления над кольцом Галуа линейной рекуррентной последовательности максимального периода над полем Галуа, Матем. вопр. криптогр., 2012, том 3, выпуск 4, 5-23

DOI: https://doi.org/10.4213/mvk65

Использование Общероссийского математического портала Math-Net.Ru подразумевает, что вы прочитали и согласны с пользовательским соглашением http://www.mathnet.ru/rus/agreement

Параметры загрузки:

IP : 54.166 .219 .16

26 апреля 2023 г., 04:58:05 
МАТЕМАТИЧЕСКИЕ ВОПРОСЫ КРИПТОГРАФИИ

2012 T. 3 № 4 C. 5-23

УДК: $511.216+519.113 .62$

Памяти В. Л. Куракина

\title{
Максимальные и минимальные представления над кольцом Галуа линейной рекуррентной последовательности максимального периода над полем Галуа
}

\author{
Р. В. Богонатов \\ ООО «Центр сертификационных исследований», Москва
}

Получено 20.V.2011

Описаны критерии максимальности и минимальности рангов представлений над кольцом Галуа линейной рекуррентной последовательности максимального периода над полем Галуа.

Ключевые слова: линейные рекуррентные последовательности, ранги представлений, кольцо Галуа, поле Галуа

To the memory of V.L. Kurakin

Maximal and minimal representations over the Galois ring for the linear recurrent maximal period sequence over the Galois field

\section{R. V. Bogonatov}

\section{LLC "Certification Research Center", Moscow}

Abstract. The representations over the Galois ring of the linear recurrent maximal period sequence over the Galois field are considered. Criteria of maximality and minimality of ranks of these representations are described.

Key words: linear recurrent sequences, ranks of representations, Galois ring, Galois field Citation: Mathematical Aspects of Cryptography, 2012, vol. 3, no. 4, pp. 5-23 (Russian). 
Пусть $R$ - коммутативное кольцо с единицей и $\sigma: \Omega \rightarrow R-$ произвольное отображение. Последовательностью $u$ над множеством $\Omega$ будем называть отображение $u: \mathbb{N}_{0} \rightarrow \Omega$, и ее $i$-й член обозначим через $u(i), i \in \mathbb{N}_{0}$. Последовательность $v=\sigma(u)$ над $R$, определяемую условием $v(i)=\sigma(u(i))$, $i \in \mathbb{N}_{0}$, назовем представлением последовательности $u$ над кольцом $R$ или $R$-представлением $u$.

Изучение представлений последовательностей дает новую информацию о свойствах последовательностей и может оказаться полезным для новых способов их использования.

Если $u-$ периодическая последовательность с длиной подхода $\lambda$ и периодом $T$, то $\sigma(u)$ - линейная рекуррентная последовательность (ЛРП) над $R$, имеющая характеристический многочлен $x^{T+\lambda}-x^{\lambda}$. Однако она может иметь и характеристические многочлены значительно меньших степеней.

В [2] Владимиром Леонидовичем Куракиным были найдены минимальные и характеристические многочлены представлений $\sigma(u)$ над кольцом вычетов $R=\mathbb{Z}_{p^{n}}$ ЛРП $u$ максимального периода над полем $P=G F(p)$. Им были описаны представления, для которых последовательность $\sigma(u)$ имеет максимальный и минимальный ранги. В [1] автором часть результатов была обобщена на случай, когда $\sigma$ отображает поле $G F\left(p^{r}\right)$ в кольцо Галуа $G R\left(p^{r n}, p^{n}\right)$. А именно: была описана каноническая система образующих аннулятора последовательности $\sigma(u)$.

В настоящей работе обобщены остальные результаты статьи [2]. Для произвольного $G R\left(p^{r n}, p^{n}\right)$-представления $\sigma$ последовательности максимального периода $u$ над $G F\left(p^{r}\right)$ показано, что ранг ЛРП $\sigma(u)$ зависит от представления $\sigma$ и ранга $M$ ЛРП $u$, но не зависит от самой ЛРП $u$. Также получены критерии максимальности и минимальности ранга таких представлений. В частности, найдены условия на отображение $\sigma$, при которых ранг последовательности $\sigma(u)$ максимален и равен

$$
1+\sum_{l=1}^{q-1}\left(d_{0}^{(l)}(M)+d_{1}^{(l)}(M)+\cdots+d_{n-1}^{(l)}(M)\right)
$$

где

$$
\begin{aligned}
& d_{c}^{(l)}(M)= \\
= & \left.\mid\left\{k \in\left\{1, \ldots, p^{r M}-2\right\}: k \equiv l \quad\left(\bmod p^{r}-1\right), w(k)=w(l)+(p-1) c\right)\right\} \mid,
\end{aligned}
$$

и через ж(t) обозначен $p$-ичный вес числа $t$. 
К сожалению, не удалось вычислить величины $d_{c}^{(l)}(M)$ и найти точное значение максимального ранга.

Пусть $R=G R\left(p^{r n}, p^{n}\right)$ - кольцо Галуа из $p^{r n}$ элементов, $P=G F\left(p^{r}\right)-$ поле из $p^{r}$ элементов и $\sigma: P \rightarrow R-$ произвольное отображение. Пусть, далее, $q=p^{r}, f(x) \in P[x]-$ многочлен максимального периода степени $M \geq 1$, $u \in L_{P}(f) \backslash\{0\}-$ линейная рекуррентная последовательность над полем $P$ максимального периода $T=p^{r M}-1$.

Будем обозначать через $\bar{a}$ образ элемента $a \in R$ при действии естественного эпиморфизма кольца $R$ в его поле вычетов $\bar{R}=R / p R=G F\left(p^{r}\right)$. Обозначим также через $\bar{f}(x)$ образ многочлена $f(x) \in P[x]$ при действии естественного эпиморфизма $P[x] \rightarrow \bar{R}[x]$. Очевидно, $\bar{f}(x)$ является многочленом максимального периода степени $M$ над полем $\bar{R}$, а значит, $\bar{f}(x) \mid x^{T}-\bar{e}$. По лемме Гензеля существует единственный унитарный многочлен $f_{*}(x) \in R[x]$, удовлетворяющий условиям

$$
f_{*}(x) \mid x^{T}-e \quad \text { и } \quad \bar{f}_{*}(x)=\bar{f}(x) .
$$

Пусть $S=G R\left(p^{M r n}, p^{n}\right)$ - расширение Галуа степени $M$ кольца Галуа $R$, содержащее корень $\theta$ многочлена $f_{*}(x)$.

Нормой $\|c\|$ элемента $c \in S$ называется величина

$$
\|c\|=\max \left\{r \in\{0, \ldots, n\}: c \in p^{r} S\right\} .
$$

Нормы представления $\sigma$ и многочлена $H(x) \in S[x]$ определим следующим образом: $\|\sigma\|=\max \left\{r \in\{0, \ldots, n\}: \sigma(a) \in p^{r} R\right.$ для всех $\left.a \in P\right\},\|H\|=$ $=\max \left\{r \in\{0, \ldots, n\}: H(x) \in p^{r} S[x]\right\}$.

Для кольца Галуа $R=G R\left(p^{r n}, p^{n}\right)$ определим $p$-адическое координатное множество $\Gamma(R)$ как множество всех корней в $R$ многочлена $x^{p^{r}}-x$ :

$$
\Gamma(R)=\left\{\beta \in R: \beta^{p^{r}}=\beta\right\} .
$$

Согласно [4, с. 128] множество $\Gamma(R)$ замкнуто относительно операции умножения и состоит из $p^{r}$ попарно не сравнимых по модулю $p R$ элементов.

Произвольный элемент $a \in R$ однозначно представляется в виде

$$
a=a_{0}+p a_{1}+\cdots+p^{n-1} a_{n-1},
$$

где $a_{0}, a_{1}, \ldots, a_{n-1} \in \Gamma(R)$. Функции $\gamma_{0}(a)=a_{0}, \ldots, \gamma_{n-1}(a)=a_{n-1}$ называются $p$-адическими координатными функциями кольца $R$.

Если на множестве $\Gamma(R)$ ввести операцию сложения $\oplus$ равенством

$$
a \oplus b=\gamma_{0}(a+b), \quad a, b \in \Gamma(R),
$$

то $(\Gamma(R), \oplus, \cdot)$ будет полем, изоморфным полю $\bar{R}=R / p R=G F\left(p^{r}\right)$. 
Рассмотрим элементы поля $P$ и $p$-адического координатного множества $\Gamma(R)$ кольца $R$ :

$$
P=\left\{a_{0}=0, a_{1}, \ldots, a_{q-1}\right\}, \quad \Gamma(R)=\left\{\beta_{0}, \ldots, \beta_{q-1}\right\}
$$

Пронумеруем элементы полей $P$ и $\Gamma(R)$ так, чтобы отображение $a_{t} \rightarrow \beta_{t}$, $t=0, \ldots, q-1$, являлось изоморфизмом.

Утверждение 1 ([1]). Сущуествует единственный многочлен

$$
\Psi_{\sigma}(x)=\psi_{q-1} x^{q-1}+\psi_{q-2} x^{q-2}+\cdots+\psi_{1} x+\psi_{0} \in R[x],
$$

для которого $\Psi_{\sigma}\left(\beta_{t}\right)=\sigma\left(a_{t}\right), t=0, \ldots, q-1$. При этом $\left\|\Psi_{\sigma}(x)\right\|=\|\sigma\|$.

Заметим, что представление $\sigma$ однозначно задается коэффициентами введенного многочлена.

Для целого числа $k \geq 0$ обозначим через $\nu_{m}(k) m$-й разряд $p$-ичного разложения $k$, а через ж(k) - сумму (в кольце целых чисел) разрядов p-ичного разложения $k$ :

$$
k=\sum_{m \geq 0} p^{m} \nu_{m}(k), \quad w(k)=\sum_{m \geq 0} \nu_{m}(k), \quad \nu_{m}(k) \in\{0, \ldots, p-1\} .
$$

Для целых чисел $k \geq 0, d \geq 1$ будем обозначать через $k \overline{\bmod } d$ число 0 , если $k=0$, и вычет числа $k$ по модулю $d$ в множестве $\{1, \ldots, d\}$, если $k \geq 1$.

В работе [1] описан минимальный многочлен последовательности $\sigma(u)$ :

$$
m_{\sigma(u)}(x)=\Delta_{n-1}(x) \prod_{\substack{1 \leq k \leq T-1 \\ t+\left\|\psi_{l}\right\| \leq n-1}}\left(x-\theta^{k}\right)
$$

где $l=k \overline{\bmod }(q-1), t=\frac{1}{p-1}(w(k)-w(l))$ и

$$
\Delta_{n-1}(x)= \begin{cases}e, & \text { если }\left\|q^{M-1} \sum_{a \in P} \sigma(a)-\sigma(0)\right\|=n, \\ x-e & \text { в противном случае. }\end{cases}
$$

Заметим, что в рассматриваемом случае, когда $u-$ ЛРП максимального периода над полем $P$, ранг ЛРП $\sigma(u)$ зависит от представления $\sigma$ и ранга $M$ ЛРП $u$ над полем $P$, но не зависит от самой ЛРП $u$. Поэтому корректно обозначение $r(\sigma, M)$ для ранга последовательности $\sigma(u)$ над $R$.

Обозначим через $\delta(\sigma, M)$ степень многочлена $\Delta_{n-1}(x), \delta(\sigma, M) \in\{0,1\}$. 


\section{Утверждение 2. Справедливо равенство}

$$
\delta(\sigma, M)= \begin{cases}0, & \text { если }\left(q^{M}-1\right) \psi_{0}+q^{M-1}(q-1) \psi_{q-1}=0, \\ 1 & \text { в противном случае. }\end{cases}
$$

Доказательство. Из определения $\Delta_{n-1}(x)$ следует, что $\delta(\sigma, M)=0$ тогда и только тогда, когда

$$
q^{M-1} \sum_{a \in P} \sigma(a)-\sigma(0)=0
$$

Верны равенства

$$
\sum_{a \in P} \sigma(a)=\sum_{\beta \in \Gamma(R)} \Psi_{\sigma}(\beta)=\sum_{\beta \in \Gamma(R)} \sum_{d=0}^{q-1} \psi_{d} \beta^{d}=\sum_{d=0}^{q-1} \psi_{d} \sum_{\beta \in \Gamma(R)} \beta^{d} .
$$

Используя формулу суммы геометрической прогрессии, несложно показать, что

$$
\sum_{\beta \in \Gamma(R)} \beta^{d}= \begin{cases}q, & \text { если } d=0, \\ q-1, & \text { если } d=q-1, \\ 0 & \text { в противном случае. }\end{cases}
$$

Тогда $\sum_{a \in P} \sigma(a)=q \psi_{0}+(q-1) \psi_{q-1}$, и условие $\delta(\sigma, M)=0$ равносильно равенству

$$
q^{M} \psi_{0}+q^{M-1}(q-1) \psi_{q-1}-\psi_{0}=0
$$

что и требовалось доказать.

В формуле (1), согласно определениям для $l$ и $t$, условие $t+\left\|\psi_{l}\right\| \leq n-1$ равносильно условию

$$
w(k)-w(l)+(p-1)|| \psi_{l} \| \leq(p-1)(n-1) .
$$

Поэтому ранг последовательности $\sigma(u)$ над $R$ вычисляется по формуле

$$
\begin{aligned}
& r(\sigma, M)= \\
& =\delta(\sigma, M)+\left|\left\{k \in\{1, \ldots, T-1\}: w(k) \leq w(l)+(p-1)\left(n-1-|| \psi_{l}||\right)\right\}\right|
\end{aligned}
$$

или

$$
\begin{gathered}
r(\sigma, M)=\delta(\sigma, M)+\sum_{l=1}^{q-1} \mid\{k \in\{1, \ldots, T-1\}: k \equiv l(\bmod q-1) \text { и } \\
\left.\omega(k) \leq \omega(l)+(p-1)\left(n-1-\| \psi_{l}||\right)\right\} \mid .
\end{gathered}
$$


Если $k \equiv l(\bmod q-1)$, то $w(k) \equiv w(l)(\bmod p-1)$, и тогда выполняется равенство

$$
\begin{gathered}
r(\sigma, M)=\delta(\sigma, M)+\sum_{l=1}^{q-1} \sum_{\substack{c=0\\
}(k)=w(l)+(p-1) c)\} \mid .}^{n-1-\psi_{l} \|} \mid\{k \in\{1, \ldots, T-1\}: k \equiv l(\bmod q-1) \text { и } \\
\end{gathered}
$$

Обозначим

$d_{c}^{(l)}(M)=\mid\{k \in\{1, \ldots, T-1\}: k \equiv l(\bmod q-1)$ и $\left.๗(k)=w(l)+(p-1) c)\right\} \mid$,

где $l \in\{1, \ldots, q-1\}$ и $c \in\{0, \ldots, r M-1\}$.

В новых обозначениях верно равенство

$$
r(\sigma, M)=\delta(\sigma, M)+\sum_{l=1}^{q-1}\left(d_{0}^{(l)}(M)+d_{1}^{(l)}(M)+\cdots+d_{n-1-|| \psi_{l}||}^{(l)}(M)\right) .
$$

В дальнейшем будет важно, какие из слагаемых $d_{c}^{(l)}(M)$ в этой сумме ненулевые.

Утверждение 3. Пусть $M \geq 2$. Тогда

a) если $l \in\{1, \ldots, q-2\}$, по $d_{c}^{(l)}(M)>0$ при $c \in\{0, \ldots,(M-1) r\}$ $u d_{c}^{(l)}(M)=0$ при с $>(M-1) r$;

б) $d_{c}^{(q-1)}(M)>0$ при $c \in\{0, \ldots,(M-2) r\}$ u $d_{c}^{(q-1)}(M)=0$ nри $c>(M-2) r$.

Доказательство. а) В работе [1] показано, что для всех $k \in\{1, \ldots, T$ $-1\}$ выполняется неравенство

$$
w(k) \leq w(l)+(p-1)(M-1) r,
$$

где $l=k \overline{\bmod }(q-1)$. Значит, если $c>(M-1) r$, то $d_{c}^{(l)}(M)=0$.

Пусть $l \in\{1, \ldots, q-2\}$ и $c \in\{0, \ldots,(M-1) r\}$. Покажем, что в этом случае $d_{c}^{(l)}(M)>0$. Положим

$D_{c}^{(l)}(M)=\{k \in\{1, \ldots, T-1\}: k \equiv l(\bmod q-1)$ и $\left.๗(k)=w(l)+(p-1) c)\right\}$.

Тогда $d_{c}^{(l)}(M)=\left|D_{c}^{(l)}(M)\right|$. 
Каждому числу $k \in\{0, \ldots, T\}$ с $p$-ичным разложением

$$
k=k_{0}+k_{1} p+\cdots+k_{r M-1} p^{r M-1}, \quad 0 \leq k_{i}<p,
$$

поставим в соответствие матрицу

$$
A(k)_{M \times r}=\left(\begin{array}{cccc}
k_{0} & k_{1} & \cdots & k_{r-1} \\
k_{r} & k_{1+r} & \cdots & k_{2 r-1} \\
\vdots & \vdots & \ddots & \vdots \\
k_{(M-1) r} & k_{1+(M-1) r} & \cdots & k_{M r-1}
\end{array}\right) .
$$

Очевидно, что заданное таким образом отображение множества $\{0,1, \ldots, T\}$ в множество матриц размера $M \times r$ с элементами, не превосходящими $p-1$, является биективным.

Введенные матрицы обладают следующими свойствами:

1)

2) $l=k \overline{\bmod }(q-1)=\left(S\left(A_{1}^{\downarrow}(k)\right)+\cdots+S\left(A_{r}^{\downarrow}(k)\right) p^{r-1}\right) \overline{\bmod }(q-1)$, где $S\left(A_{i}^{\downarrow}(k)\right)$ - сумма элементов $i$-го столбца матрицы $A(k), i \in\{1, \ldots, r\}$.

3) $0 \leq S\left(A_{i}^{\downarrow}(k)\right) \leq(p-1) M$ для каждого $i \in\{1, \ldots, r\}$.

Докажем, что $d_{c}^{(l)}(M)>0$ при $c \in\{0, \ldots,(M-1) r\}$ и $l \in\{1, \ldots, q-2\}$, индукцией по $c$ при фиксированном $l$.

Пусть $l \in\{1, \ldots, q-2\}$ и $c=0$. Тогда $d_{0}^{(l)}>0$, так как $l \in D_{0}^{(l)}(M)$.

Пусть теперь $d_{c}^{(l)}(M)>0$ для некоторого $c \in\{0, \ldots,(M-1) r-1\}$. Покажем, что тогда $d_{c+1}^{(l)}(M)>0$. Действительно, по предположению индукции существует такое $k \in D_{c}^{(l)}(M)$, что

$$
k \equiv l(\bmod q-1), \quad w(k)=w(l)+(p-1) c .
$$

Докажем, что в матрице $A(k)$ найдется такой столбец $A_{i}^{\downarrow}(k)$, что

$$
S\left(A_{i}^{\downarrow}(k)\right) \leq(p-1) M-p .
$$

Предположим противное. Тогда для каждого $j \in\{1, \ldots, r\}$ выполняется неравенство

$$
S\left(A_{j}^{\downarrow}(k)\right) \geq(p-1) M-p+1=(p-1)(M-1),
$$

т. е. существует такое $t_{j} \in\{0, \ldots, p-1\}$, что

$$
S\left(A_{j}^{\downarrow}(k)\right)=(p-1)(M-1)+t_{j} .
$$


Далее, по свойствам матрицы $A(k)$ выполняется цепочка сравнений по модулю $q-1$

$$
\begin{gathered}
l \equiv k \equiv \sum_{j=0}^{r-1} S\left(A_{j+1}^{\downarrow}(k)\right) p^{j} \equiv \sum_{j=0}^{r-1}\left((p-1)(M-1)+t_{j+1}\right) p^{j} \equiv \\
\equiv(M-1) \sum_{j=0}^{r-1}(p-1) p^{j}+\sum_{j=0}^{r-1} t_{j+1} p^{j} \equiv(M-1)(q-1)+\sum_{j=0}^{r-1} t_{j+1} p^{j} \equiv \sum_{j=0}^{r-1} t_{j+1} p^{j}, \\
\text { а значит, } l=\sum_{j=0}^{r-1} t_{j+1} p^{j} . \text { При этом } \\
w(k)=\sum_{j=1}^{r} S\left(A_{j}^{\downarrow}(k)\right)=\sum_{j=1}^{r}\left((p-1)(M-1)+t_{j}\right)= \\
=(p-1)(M-1) r+\sum_{j=1}^{r} t_{j}=w(l)+(p-1)(M-1) r .
\end{gathered}
$$

С другой стороны, по определению числа $k$

$$
w(k)=w(l)+(p-1) c,
$$

т. е. $c=(M-1) r$, что противоречит выбору $c$. Таким образом, доказано существование в матрице $A(k)$ столбца со свойством (4).

Теперь можно выбрать такое $i \in\{1, \ldots, r\}$, что

$$
S\left(A_{i}^{\downarrow}(k)\right) \leq(p-1) M-p, \text { a } S\left(A_{i+1}^{\downarrow}(k)\right)>0
$$

(или $S\left(A_{1}^{\downarrow}(k)\right)>0$ для $i=r$ ). Очевидно, что существует матрица $A\left(k^{\prime}\right)$, для которой

$$
S\left(A_{i}^{\downarrow}\left(k^{\prime}\right)\right)=S\left(A_{i}^{\downarrow}(k)\right)+p, \text { a } S\left(A_{i+1}^{\downarrow}\left(k^{\prime}\right)\right)=S\left(A_{i+1}^{\downarrow}(k)\right)-1
$$

(или $S\left(A_{1}^{\downarrow}\left(k^{\prime}\right)\right)=S\left(A_{1}^{\downarrow}(k)\right)-1$ для $i=r$ ), а все остальные столбцы совпадают со столбцами матрицы $A(k)$.

Несложно показать, что для числа $k^{\prime}$, которое задает матрица $A\left(k^{\prime}\right)$, верны соотношения

$$
k^{\prime} \equiv k \equiv l(\bmod q-1), \quad w\left(k^{\prime}\right)=w(k)+(p-1)=w(l)+(p-1)(c+1),
$$

т. е. $k^{\prime} \in D_{c+1}^{(l)}(M)$ и $d_{c+1}^{(l)}(M)>0$, что и требовалось доказать. 
б) Пусть $c \in\{0, \ldots,(M-2) r\}$. Доказательство того, что $d_{c}^{(q-1)}(M)>0$, можно провести индукцией по $c$ аналогично тому, как это было сделано в пункте а). Различие будет лишь в обосновании неравенства (4): для $c \in$ $\in\{0, \ldots,(M-2) r-1\}$ и $k \in D_{c}^{(q-1)}(M)$ в матрице $A(k)$ найдется столбец, в котором сумма элементов не превосходит величины $(p-1) M-p$. Докажем это. Предположим противное, тогда

$$
w(k)=\sum_{i=1}^{r} S\left(A_{i}^{\downarrow}(k)\right) \geq((p-1) M-p+1) r=(p-1)(M-1) r .
$$

Но так как $k \in D_{c}^{(q-1)}(M)$, то $w(k)=r(p-1)+(p-1) c$. Получили неравенство

$$
(p-1) r+(p-1) c \geq(p-1)(M-1) r,
$$

из которого следует, что $c \geq(M-2) r$, что противоречит выбору числа $c$.

Пусть теперь $c>(M-2) r$. Покажем, что $d_{c}^{(q-1)}(M)=0$. Действительно, пусть $k \in D_{c}^{(q-1)}(M)$, тогда выполняются соотношения

$$
k \equiv 0(\bmod q-1), \quad \omega(k)=(p-1) r+(p-1) c .
$$

Так как $c>(M-2) r$, то $w(k)>(p-1)(M-1) r$.

Рассмотрим число $k^{\prime}=T-k=p^{r M}-1-k$. Очевидно, что

$$
w\left(k^{\prime}\right)=w(T)-w(k)<r M(p-1)-(p-1)(M-1) r=(p-1) r .
$$

С другой стороны, заметим, что $k^{\prime} \equiv 0(\bmod q-1)$. Если бы выполнялось неравенство $k^{\prime} \geq 1$, то из [1] следовало бы, что $w\left(k^{\prime}\right) \geq(p-1) r$. Значит, $k^{\prime}=0$ и $k=T$, и мы получили противоречие, так как $T \notin D_{c}^{(q-1)}(M)$.

Опишем представления $\sigma$, для которых ранг $r(\sigma, M)$ ЛРП $\sigma(u)$ принимает наибольшее при заданном $M$ значение.

Обозначим

$$
R(M)=1+\sum_{l=1}^{q-1}\left(d_{0}^{(l)}(M)+d_{1}^{(l)}(M)+\cdots+d_{n-1}^{(l)}(M)\right) .
$$

Очевидно, что для любого представления $\sigma$ выполняется неравенство $r(\sigma, M) \leq R(M)$ (см. (2)).

Представление $\sigma$ назовем $M$-максимальным, если $r(\sigma, M)=R(M)$, и максимальным, если оно $M$-максимально для любого $M \geq 2$. 
Теорема 1. а) Если $2 \leq M \leq \frac{n+r-1}{r}$, то представление $\sigma$ М-максимально тогда и только тогда, когда $\left\|\psi_{l}\right\| \leq n-1-(M-1) r$ при $l \in\{1, \ldots, q-2\},\left\|\psi_{q-1}\right\| \leq n-1-(M-2) r u\left(q^{M}-1\right) \psi_{0}+q^{M-1}(q-1) \psi_{q-1} \neq 0$.

б) Если $\frac{n+r-1}{r}<M<\frac{n+2 r-1}{r}$, то представление $\sigma$ М-максимально тогда и только тогда, когда $\left\|\psi_{1}\right\|=\cdots=\left\|\psi_{q-2}\right\|=0,\left\|\psi_{q-1}\right\| \leq n-1-(M-$ 2) $r u\left(q^{M}-1\right) \psi_{0}+q^{M-1}(q-1) \psi_{q-1} \neq 0$.

в) Если $M \geq \frac{n+2 r-1}{r}$, то представление $\sigma$ М-максимально тогда и только тогда, когда

$$
\left\|\psi_{1}\right\|=\cdots=\left\|\psi_{q-1}\right\|=0 \text { u } \psi_{0} \neq 0
$$

г) Представление б максимально тогда и только тогда, когда

$$
\left\|\psi_{1}\right\|=\cdots=\left\|\psi_{q-1}\right\|=0 u\left(q^{M}-1\right) \psi_{0}+q^{M-1}(q-1) \psi_{q-1} \neq 0 .
$$

Доказательство. а) Пусть $2 \leq M \leq \frac{n+r-1}{r}$, тогда $n-1 \geq(M-1) r>$ $(M-2) r$. В этом случае, согласно утверждению 3 , в правой части равенства (5) слагаемые $d_{c}^{(l)}(M)$ равны нулю при $c \in\{(M-1) r+1, \ldots, n-1\}$ для $l \in\{1, \ldots, q-2\}$ и при $c \in\{(M-2) r+1, \ldots, n-1\}$ для $l=q-1$. Отсюда следует, что

$$
\begin{aligned}
R(M) & =1+\sum_{l=1}^{q-2}\left(d_{0}^{(l)}(M)+d_{1}^{(l)}(M)+\cdots+d_{r(M-1)}^{(l)}(M)\right)+ \\
& +\left(d_{0}^{(q-1)}(M)+d_{1}^{(q-1)}(M)+\cdots+d_{(M-2) r}^{(q-1)}(M)\right),
\end{aligned}
$$

причем все слагаемые в этой сумме положительны. Поэтому ввиду (2) $r(\sigma, m)=R(M)$ тогда и только тогда, когда $n-1-\left\|\psi_{l}\right\| \geq(M-1) r$ при $l \in\{1, \ldots, q-2\}, n-1-\left\|\psi_{q-1}\right\| \geq(M-2) r$ и $\delta(\sigma, M)=1$. По утверждению 2 последнее равенство верно тогда и только тогда, когда

$$
\left(q^{M}-1\right) \psi_{0}+q^{M-1}(q-1) \psi_{q-1} \neq 0
$$

в) Пусть $M \geq \frac{n+2 r-1}{r}$, тогда $n-1 \leq(M-2) r<(M-1) r$. Из утверждения 3 вытекает, что все слагаемые в сумме (5) положительны. Поэтому в силу (2) равенство $r(\sigma, m)=R(M)$ выполняется тогда и только тогда, когда $\left\|\psi_{1}\right\|=\cdots=\left\|\psi_{q-1}\right\|=0$ и $\delta(\sigma, M)=1$. Из неравенства $M \geq \frac{n+2 r-1}{r}$ получим неравенство $(M-1) r \geq n+r-1 \geq n$. Тогда из утверждения 2 следует, что условие $\delta(\sigma, M)=1$ равносильно условию $\psi_{0} \neq 0$. 
б) Пусть $\frac{n+r-1}{r}<M<\frac{n+2 r-1}{r}$. Тогда $(M-2) r<n-1<(M-1) r$. Далее доказательство аналогично пунктам а) и в).

г) Следует из а), б) и в).

ПримЕР. Пусть $b \in R \backslash p R$. Зададим отображение $\sigma$ правилом

$$
\sigma(a)= \begin{cases}b, & a \in P \backslash e_{P}, \\ q b, & a=e_{P} .\end{cases}
$$

Непосредственно проверяется, что $\Psi_{\sigma}(x)=b\left(x^{q-1}+\cdots+x+e\right)$. Следовательно, по теореме 1 представление $\sigma$ максимально.

ЗАМЕчАНИЕ 1 . При $r=1$ теорема 1 была доказана В. Л. Куракиным в [2].

В связи с полученными результатами представляет интерес оценить величину $R(M)$.

Обозначим через $\left\{\begin{array}{l}m \\ k\end{array}\right\}_{p}$ количество размещений $k$ одинаковых предметов в $m$ различных ящиках при условии, что в каждый ящик попадает не более $p-1$ предметов. Эта величина, согласно [5], равна

$$
\begin{aligned}
& \left\{\begin{array}{l}
m \\
k
\end{array}\right\}_{p}=\sum_{j \geq 0}(-1)^{j}\left(\begin{array}{c}
m \\
j
\end{array}\right)\left(\begin{array}{c}
k+m-p j-1 \\
m-1
\end{array}\right), \quad 1 \leq k \leq(p-1) m, \\
& \left\{\begin{array}{l}
m \\
k
\end{array}\right\}_{p}=0, \quad k>(p-1) m .
\end{aligned}
$$

При фиксированных $k$ и $p$ при $m \rightarrow \infty$ выполняются асимптотические соотношения

$$
\left\{\begin{array}{c}
m \\
k
\end{array}\right\}_{p} \sim\left(\begin{array}{c}
m \\
k
\end{array}\right) \sim \frac{m^{k}}{k !} .
$$

Отметим, что при $p=2$ выполняется равенство $\left\{\begin{array}{l}m \\ k\end{array}\right\}_{p}=\left(\begin{array}{c}m \\ k\end{array}\right)$.

Утверждение 4. а) Если $M \leq \frac{n+r-1}{r}$, $\operatorname{mo} R(M)=T=p^{r M}-1$.

б) Если $M>\frac{n+r-1}{r}$, то для $R(M)$ выполняются оценки

$$
1+\sum_{k=1}^{n(p-1)}\left\{\begin{array}{c}
r M \\
k
\end{array}\right\}_{p} \leq R(M) \leq 1+\sum_{k=1}^{(n+r-1)(p-1)}\left\{\begin{array}{c}
r M \\
k
\end{array}\right\}_{p}<T
$$


Доказательство. а) Обозначим для $t \geq 1$

$$
Q_{t}=\{k \in\{1, \ldots, T-1\}: w(k) \leq t\} .
$$

Заметим, что количество чисел множества $\left\{1, \ldots, p^{r M}-1\right\}$, имеющих вес $k \geq 1$, согласно определению (6), равно $\left\{\begin{array}{c}r M \\ k\end{array}\right\}_{p}$. Тогда $\left|Q_{t}\right|=\sum_{k=1}^{t}\left\{\begin{array}{c}r M \\ k\end{array}\right\}_{p}$.

Так как $M \leq \frac{n+r-1}{r}$ (или $\left.(M-1) r \leq n-1\right)$, то из утверждения 3 и равенства (5) следует, что $R(M)=1+\left|Q_{r M(p-1)}\right|$. Осталось заметить, что для любого $k \in\{1, \ldots, T-1\}$ выполняется неравенство

б) Зададим множество

$$
Q=\{k \in\{1, \ldots, T-1\}: \exists l \in\{1, \ldots, q-1\},
$$

$\exists c \in\{0, \ldots, n-1\}: k \equiv l(\bmod q-1)$ и $ш(k)=w(l)+(p-1) c)\}$.

Согласно условию $(M-1) r>n-1$ и равенству (5), выполняется равенство $R(M)=1+|Q|$.

Таким образом, для доказательства утверждения 4 необходимо доказать включения

$$
Q_{n(p-1)} \subset Q \subset Q_{(n+r-1)(p-1)} .
$$

Пусть $k \in Q_{n(p-1)}$. Возьмем $l=k \overline{\bmod }(q-1)$. Тогда $w(l)>0$ и $-w(l)<n(p-1)$. Так как

$$
w(k)-w(l)=(p-1) c, \quad \text { где } c \leq n-1 .
$$

Следовательно, $k \in Q$.

Если же $k \in Q$, то

$$
w(k)=w(l)+c(p-1) \leq(p-1) r+(n-1)(p-1)=(r+n-1)(p-1),
$$

и $k \in Q_{(n+r-1)(p-1)}$.

ЗАМЕчАНИЕ 2. При $r=1$ двойное неравенство в П. б) утверждения 4 превращается в равенство

$$
R(M)=1+\sum_{k=1}^{n(p-1)}\left\{\begin{array}{c}
M \\
k
\end{array}\right\}_{p}<T
$$

которое было доказано в [2]. 
Представление $\sigma$ назовем собственным, если $\sigma \neq$ const $(\bmod p R)$, и несобственным в противном случае. Всякое представление есть сумма $\sigma=\widetilde{\sigma}+\sigma_{0}$, где $\sigma_{0}-$ представление-константа, определяемое равенствами $\sigma_{0}(a)=\sigma(0), a \in P$. Если представление $\sigma$ несобственное, то $\|\widetilde{\sigma}\|>0$, т. е. $\widetilde{\sigma}: P \rightarrow p R$, и $\widetilde{\sigma}$ можно рассматривать как представление над кольцом $G R\left(p^{r(n-1)}, p^{n-1}\right)$, продолжив его с помощью отображения $\tau: p R \rightarrow G R\left(p^{r(n-1)}, p^{n-1}\right)$, определяемого следующим образом:

$$
\tau\left(a_{1} p+\cdots+a_{n-1} p^{n-1}\right)=a_{1}+\cdots+a_{n-1} p^{n-2},
$$

где $a_{i}-p$-адические координаты элемента множества $p R$. При этом не утрачиваются особенности исходного представления $\widetilde{\sigma}$, поскольку по $\tau(\widetilde{\sigma})$ можно однозначно восстановить $\widetilde{\sigma}$. Таким образом, несобственное представление отличается на константу от представления $\widetilde{\sigma}$, которое можно рассматривать как представление над кольцом $G R\left(p^{r(n-1)}, p^{n-1}\right)$ меньшей характеристики $p^{n-1}$. По этой причине несобственные представления устроены в некотором смысле проще, чем собственные. Поэтому далее при определении минимального представления рассматриваются только собственные представления.

Обозначим

$$
r(M)= \begin{cases}q+q^{2}+\cdots+q^{M-1}, & \text { если } M \leq \frac{n+r-1}{r}, \\ d_{0}^{(1)}(M)+\cdots+d_{n-1}^{(1)}(M), & \text { если } M>\frac{n+r-1}{r} .\end{cases}
$$

Согласно этому определению, $r(1)=0$. Собственное представление $\sigma$ назовем $M$-минимальным, если $r(\sigma, M)=r(M)$.

Теорема 2. Пусть представление $\sigma$ - собственное. Тогда для $M \geq 1$ справедливо неравенство $r(\sigma, M) \geq r(M)$.

Представление $\sigma$ тогда и только тогда 1-минимально, когда

$$
\overline{\sigma(0)} \neq \overline{0}, \quad \sigma(a)=0, \quad a \in P \backslash 0 .
$$

Если $2 \leq M \leq \frac{n+r-1}{r}$, то б тогда и только тогда $M$-минимально, когда существует такое $b \in R \backslash p R$, что

$$
\sigma(a)=b, \quad a \in P \backslash 0, \quad \sigma(0)=-q^{M-1} \frac{q-1}{q^{M-1}-1} \cdot b .
$$


Если $M>\frac{n+r-1}{r}$, то $\sigma$ тогда и только тогда М-минимально, когда для подходящих $b \in R \backslash p R$ и $s \in\{0, \ldots, r-1\}$ выполняется равенство $\Psi_{\sigma}(x)=b x^{p^{s}}$, m.e.

$$
\sigma(a)=\Psi_{\sigma}\left(\beta_{a}\right)=b \beta_{a}^{p^{s}}, \quad a \in P
$$

( $\beta_{a}$ - образ элемента а при заданном выше изоморфизме поля $P$ в поле $\left.\Gamma(R)\right)$.

Доказательство. Для $l \in\{1, \ldots, q-1\}$ обозначим

$$
Q_{c}^{(l)}(M)=d_{0}^{(l)}(M)+\cdots+d_{c}^{(l)}(M) .
$$

Заметим, что $\|\widetilde{\sigma}\|=0$, поскольку $\sigma$ собственное, и по утверждению 1 $\left\|\Psi_{\widetilde{\sigma}}(x)\right\|=0$. Но $\Psi_{\widetilde{\sigma}}(x)=\Psi_{\sigma}(x)-\sigma(0)=\Psi_{\sigma}(x)-\psi_{0}$, и, следовательно,

$$
\left\|\psi_{l}\right\|=0 \quad \text { для некоторого } l \in\{1, \ldots, q-1\} \text {. }
$$

Таким образом, согласно (2)

$$
r(\sigma, M) \geq \delta(\sigma, M)+\min _{\substack{1 \leq l \leq q-1 \\\left\|\psi_{l}\right\|=0}} Q_{n-1}^{(l)}(M) \geq \min _{\substack{1 \leq l \leq q-1 \\\left\|\psi_{l}\right\|=0}} Q_{n-1}^{(l)}(M) .
$$

a) Рассмотрим случай $M \leq \frac{n+r-1}{r}$. Докажем неравенство $r(\sigma, M) \geq$ $\geq r(M)$. Для этого вычислим $Q_{n-1}^{(l)}(M)$. Заметим, что ввиду утверждения 3 при $M \leq \frac{n+r-1}{r}$ (т. е. при $\left.r(M-1) \leq n-1\right)$ верно равенство

$$
Q_{(n-1)}^{(l)}(M)=\sum_{c=0}^{\infty} d_{c}^{(l)}(M) .
$$

Из определения $d_{c}^{(l)}(M)$ и последнего равенства несложно вывести, что величина $Q_{(n-1)}^{(l)}(M)$ равна количеству таких чисел $k \in\{1, \ldots, T-1\}$, что $k \equiv l(\bmod q-1)$. А именно,

$$
Q_{(n-1)}^{(l)}(M)= \begin{cases}T /(q-1)=r(M)+1, & \text { если } l \in\{1, \ldots, q-2\}, \\ T /(q-1)-1=r(M), & \text { если } l=q-1 .\end{cases}
$$

Неравенство $r(\sigma, M) \geq r(M)$ вытекает теперь из (9).

Выясним, какие представления $M$-минимальны. В силу (2), (9) и (10) неравенство $r(\sigma, M) \geq r(M)$ обращается в равенство тогда и только тогда, когда $l=q-1$ и в сумме (2) все слагаемые равны нулю, за исключением 
слагаемых при $l=q-1$. Так как представление $\sigma$ собственное, то это равносильно соотношениям

$$
\psi_{1}=\cdots=\psi_{q-2}=0, \quad\left\|\psi_{q-1}\right\|=0, \quad \delta(\sigma, M)=0 .
$$

Тогда $\Psi_{\sigma}(x)=\psi_{q-1} x^{q-1}+\psi_{0}$, откуда $\sigma(0)=\psi_{0}, \sigma(a)=\Psi_{\sigma}\left(\beta_{a}\right)=\psi_{q-1}+\psi_{0}$, где $a \in P$, а $\beta_{a}-$ образ элемента $a$ при заданном выше изоморфизме поля $P$ в поле $\Gamma(R)$. Обозначим $\psi_{q-1}+\psi_{0}$ через $b$. Тогда условия (11) примут вид

$$
\sigma(a)=b, a \in P \backslash 0, \quad \sigma(0)=b-\psi_{q-1}, \quad\left\|\psi_{q-1}\right\|=0, \quad \delta(\sigma, M)=0 .
$$

Ввиду утверждения 2 равенство $\delta(\sigma, M)=0$ эквивалентно соотношению $b-\psi_{q-1}=q^{M-1}\left(q b-\psi_{q-1}\right)$. Окончательно получаем, что $\sigma$ тогда и только тогда $M$-минимально, когда

$$
\begin{gathered}
\sigma(a)=b, a \in P \backslash 0, \quad \sigma(0)=b-\psi_{q-1}, \\
\left\|\psi_{q-1}\right\|=0, \quad b-\psi_{q-1}=q^{M-1}\left(q b-\psi_{q-1}\right) .
\end{gathered}
$$

Если $M=1$, то $b=0$ и $\overline{\sigma(0)}=-\overline{\psi_{q-1}} \neq \overline{0}$, что и требовалось доказать.

Если $2 \leq M \leq \frac{n+r-1}{r}$, то из (12) находим, что $\psi_{q-1}=\frac{q^{M}-1}{q^{M-1}-1} \cdot b$, откуда

$$
\sigma(0)=b-\psi_{q-1}=-q^{M-1} \frac{q-1}{q^{M-1}-1} \cdot b .
$$

При этом $\bar{b} \neq \overline{0}$, поскольку $\left\|\psi_{q-1}\right\|=0$.

б) Пусть теперь $M>\frac{n+r-1}{r}$. Докажем предварительно две леммы.

Лемма 1. Пусть $l_{1} \in\{1, \ldots, q-1\} u l_{2}=p^{s} l_{1} \overline{\bmod }(q-1)$, $s \in\{1, \ldots, n-1\}$, т.е. $l_{2}$ получается из $l_{1}$ ииклическим сдвигом р-ичных координат. Тогда $d_{c}^{\left(l_{1}\right)}(M)=d_{c}^{\left(l_{2}\right)}(M)$ для любого $c \geq 0$.

Доказательство. Очевидно, что $w\left(l_{1}\right)=w\left(l_{2}\right)$.

Рассмотрим отображение $\varphi: D_{c}^{\left(l_{1}\right)}(M) \rightarrow\{1, \ldots, T\}$, заданное по правилу $\varphi(k)=p^{s} k \overline{\bmod } T$ (множество $D_{c}^{\left(l_{1}\right)}(M)$ определено в (3)). Докажем, что $\varphi-$ биекция на множество $D_{c}^{\left(l_{2}\right)}(M)$. Действительно, пусть $k \in D_{c}^{\left(l_{1}\right)}(M)$, т. е.

$$
k \equiv l_{1}(\bmod q-1), \quad w(k)=w\left(l_{1}\right)+(p-1) c .
$$

Заметим, что $\varphi(k) \equiv p^{s} l_{1}(\bmod q-1)$ и, значит, $\varphi(k) \equiv l_{2}(\bmod q-1)$. Так как $\varphi$ - циклический сдвиг $p$-ичных координат, то

$$
w(\varphi(k))=w(k)=w\left(l_{1}\right)+(p-1) c=w\left(l_{2}\right)+(p-1) c,
$$


и $\varphi(k) \in D_{c}^{\left(l_{2}\right)}(M)$. Мы доказали, что $\varphi\left(D_{c}^{\left(l_{1}\right)}(M)\right) \subset D_{c}^{\left(l_{2}\right)}(M)$. Несложно заметить, что отображение $\varphi$ инъективно и сюръективно, а значит, и биективно. Следовательно, $\left|D_{c}^{\left(l_{1}\right)}(M)\right|=\left|D_{c}^{\left(l_{2}\right)}(M)\right|$, или $d_{c}^{\left(l_{1}\right)}(M)=d_{c}^{\left(l_{2}\right)}(M)$.

Лемма 2. Пусть $M>\frac{n+r-1}{r}, l \in\{1, \ldots, q-1\} u w(l)>1$. Тогда

$$
Q_{(n-1)}^{(l)}(M)>Q_{(n-1)}^{(1)}(M) .
$$

Доказательство. Напомним, что

$$
Q_{n-1}^{(l)}(M)=d_{0}^{(l)}(M)+\cdots+d_{n-1}^{(l)}(M) .
$$

Из леммы 1 следует равенство $Q_{n-1}^{(l)}(M)=Q_{n-1}^{\left(l^{\prime}\right)}(M)$, где $l^{\prime}$ получается из $l$ циклическим сдвигом $p$-ичных координат. Поэтому без ограничения общности будем считать, что для числа $l$ выполняется неравенство $l_{0}>0$.

Зададим отображение $\varphi: \bigcup_{c=0}^{n-1} D_{c}^{(1)}(M) \rightarrow \bigcup_{c=0}^{n-1} D_{c}^{(l)}(M)$ правилом $\varphi(k)=$ $k+l-1$. Докажем корректность заданного отображения. Действительно, пусть $k \in \bigcup_{c=0}^{n-1} D_{c}^{(1)}(M)$, тогда

$k \in\{1, \ldots, T-1\}, \quad k \equiv 1(\bmod q-1), \quad w(k)=1+(p-1) c, c \leq n-1$.

Из выписанных условий следует, что либо $k=T-q+2$, либо $k \leq T-2 q+3$. В первом случае

$$
w(k)=(p-1) r M-(p-1) r+1=1+(p-1)(M-1) r,
$$

что противоречит условию $M>\frac{n+r-1}{r}$ (или $\left.(M-1) r>n-1\right)$. Во втором случае, очевидно, $\varphi(k)=k+l-1 \leq T-1$. Далее, из условия $k \equiv 1(\bmod q-1)$ следует, что $\varphi(k) \equiv l(\bmod q-1)$. Найдем

$$
w(\varphi(k))=w(k)+w(l-1)-(p-1) c^{\prime},
$$

где $c^{\prime}-$ число переносов, возникающих при сложении чисел $k$ и $(l-1)$. Учитывая, что $l_{0}>0$ и, значит,

$$
w(\varphi(k))=1+(p-1) c+w(l)-1-(p-1) c^{\prime}=w(l)+(p-1)\left(c-c^{\prime}\right) .
$$

Следовательно, $๗(\varphi(k)) \in \bigcup_{c=0}^{n-1} D_{c}^{(l)}(M)$, и отображение $\varphi$ задано корректно. 
Для доказательства леммы достаточно показать, что введенное отображение $\varphi$ инъективно, но не сюръективно. Инъективность очевидна. Докажем, что отображение $\varphi$ не сюръективно. Пусть число $i \in\{0, \ldots, r-1\}$ максимальное со свойством $l_{i} \neq 0$. Рассмотрим число $k$ с $p$-ичным разложением

$$
k=1+\left(p-l_{i}\right) p^{i}+(p-1)\left(p^{i+1}+\cdots+p^{(M-1) r+i-1}\right)+\left(l_{i}-1\right) p^{(M-1) r+i} .
$$

Определение корректно и при $i=0$, так как по условию $w(l)>1$ и, значит, $l_{0}>1$.

Несложно показать, что $k \equiv 1(\bmod q-1)$ и $(k)=1+(p-1)(M-1) r$. Так как по условию $(M-1) r>n-1$, то $k \notin \bigcup_{c=0}^{n-1} D_{c}^{(1)}(M)$.

Рассмотрим теперь число $k^{\prime}=k+l-1$. Легко проверить, что $k^{\prime} \equiv l(\bmod q-1)$ и $k^{\prime}$ имеет $p$-ичное разложение

$$
k^{\prime}=l_{0}+l_{1} p+\cdots+l_{i-1} p^{i-1}+l_{i} p^{(M-1) r+i} .
$$

Значит, $\in \bigcup_{c=0}^{n-1} D_{c}^{(l)}(M)$ нет прообраза в $\bigcup_{c=0}^{n-1} D_{c}^{(1)}(M)$ при отображении $\varphi$. Следовательно, $\varphi$ не сюръективно.

Вернемся к доказательству теоремы 2. Пользуясь леммой 2, продолжим неравенство (9):

$$
r(\sigma, M) \geq \min _{\substack{1 \leq l \leq q-1 \\\left\|\psi_{l}\right\|=0}} Q_{n-1}^{(l)}(M) \geq Q_{n-1}^{(1)}(M)=r(M) .
$$

Здесь $Q_{n-1}^{(1)}(M)=r(M)$ в соответствии с определениями (7), (8). Таким образом, неравенство $r(\sigma, M) \geq r(M)$ доказано.

Опишем теперь $M$-минимальные представления. В силу (2), (13), леммы 1 и леммы 2 равенство $r(\sigma, M)=r(M)$ выполняется тогда и только тогда, когда существует такое $l \in\{1, \ldots, q-1\}$, что

$$
\omega(l)=1, \quad\left\|\psi_{l}\right\|=0, \quad \psi_{l^{\prime}}=0, \quad l^{\prime} \in\{1, \ldots, q-1\} \backslash\{l\}, \quad \delta(\sigma, M)=0 .
$$

Так как $M>\frac{n+r-1}{r}$, т. е. $r M>n+r-1 \geq n$, то, согласно утверждению 2 , равенство $\delta(\sigma, M)=0$ эквивалентно тому, что $\psi_{0}=0$. Тогда $\Psi_{\delta}(x)=b x^{l}$ для 
некоторого $b \in R \backslash p R$ и $(l)=1$. Так как равенство тому, что $l=p^{s}$ для некоторого $s \in\{0, \ldots, r-1\}$, то $\Psi_{\sigma}(x)=b x^{p^{s}}$, т. е.

$$
\sigma(a)=\Psi_{\sigma}\left(\beta_{a}\right)=b \beta_{a}^{p^{s}}, \quad a \in P .
$$

Теорема 2 доказана.

Следствие 1. Если $\sigma$ является $M$-минимальным и $1 \leq M \leq \frac{n+r-1}{r}$, то $\sigma(0) \neq 0$. Если $\sigma$ является $М$-минимальным и $M>\frac{n+r-1}{r}$, то $\sigma(0)=0$.

Собственное представление $\sigma$ будем называть минимальным, если оно $M$-минимально для любого $M>\frac{n+r-1}{r}$. Отметим, что ввиду следствия теоремы 2 не существует представлений, $M$-минимальных для всех $M \geq 1$.

Утверждение 5. Представление $\sigma$ минимально тогда и только тогда, когда существуют такие $b \in R \backslash p R u s \in\{0, \ldots, r-1\}$, что выполняется равенство $\Psi_{\sigma}(x)=b x^{p^{s}}$, m.e.

$$
\sigma(a)=\Psi_{\sigma}\left(\beta_{a}\right)=b \beta_{a}^{p^{s}}, \quad a \in P .
$$

Если б минимально и $M \leq \frac{n+r-1}{r}$, по $r(\sigma, M)=r(M)+1$.

Доказательство. Первая часть данного утверждения вытекает из теоремы 2. Пусть $\sigma$ минимально и $M \leq \frac{n+r-1}{r}$. По доказанному $\Psi_{\sigma}(x)=b x^{p^{s}}$ для подходящих $b$ и $s$. Пользуясь соотношениями (2), (8) и (10), находим, что

$$
\begin{gathered}
r(\sigma, M)=\delta(\sigma, M)+d_{0}^{(1)}(M)+\cdots+d_{n-1}^{(1)}(M)= \\
=\delta(\sigma, M)+Q_{n-1}^{(1)}(M)=\delta(\sigma, M)+r(M)+ \begin{cases}1, & \text { если } p^{r}>2, \\
0, & \text { если } p^{r}=2 .\end{cases}
\end{gathered}
$$

В соответствии с утверждением 2

$$
\delta(\sigma, M)= \begin{cases}0, & \text { если } p^{r}>2 \\ 1, & \text { если } p^{r}=2 .\end{cases}
$$

Следовательно, $r(\sigma, M)=r(M)+1$. 


\section{Список литературы}

1. Богонатов Р. В. Представление над кольцом Галуа линейной рекуррентной последовательности над полем Галуа // Дискретная математика. 2007. - Т. 19. Вып. 1. - С. 141-157.

2. Куракин В.Л. Представления над кольцом $\mathbb{Z}_{p^{n}}$ линейной рекуррентной последовательности максимального периода над полем $G F(p)$ // Дискретная математика. - 1992. - Т. 4. Вып. 4. - С. 96-116.

3. Лидл Р., Нидеррайтер Г. Конечные поля. - М.: Мир, 1988. - 818 с.

4. Нечаев А. А. Код Кердока в циклической форме // Дискретная математика. - 1989. - Т. 1. Вып. 4. - С. 123-139.

5. Сачков В.Н. Введение в комбинаторные методы дискретной математики. - М.: Наука, 1982. - 384 с. 